\title{
Cerebellar Modules: Individual or Composite Entities?
}

\author{
Nadia L. Cerminara \\ School of Physiology and Pharmacology, University of Bristol, Medical Sciences Building, University of Bristol, Bristol BS8 1TD, United Kingdom \\ Review of Horn et al.
}

It has long been known that the cerebellum is vital for motor control, enabling movements to be performed smoothly, rapidly, and accurately. A key feature of cerebellar organization is its division into a series of smaller anatomical/functional units called modules. Each module consists of a longitudinal strip or zone of Purkinje cells within the cerebellar cortex and is defined by its climbing input originating from a circumscribed subdivision of the inferior olivary complex. Purkinje cells within each zone have similar receptive field characteristics and project to common groups of cells in the cerebellar and vestibular nuclei. In turn, efferent neurons from these nuclei are directed to motor cell groups belonging to the medial and lateral descending motor pathways, thereby directly influencing activity in descending motor pathways.

Given their defining features, cerebellar modules are thought to represent individual functional entities and may be thought of as the cerebellar counterparts of cerebral cortical columns (for review, see Apps and Garwicz, 2005). However, although much is known about the anatomical wiring of cerebellar modules, the role that each module plays in ensuring that movements are performed smoothly and accurately remains unknown.

\footnotetext{
Received Sept. 14, 2010; revised 0ct. 11, 2010; accepted 0ct. 13, 2010. I acknowledge the support of the Wellcome Trust.

Correspondence should be addressed to Nadia L. Cerminara School of Physiology and Pharmacology, University of Bristol, Medical Sciences Building, University of Bristol, Bristol BS8 1TD, UK. E-mail: n.cerminara@bristol.ac.uk.

DOI:10.1523/JNEUROSCI.4823-10.2010

Copyright $\odot 2010$ the authors $\quad 0270-6474 / 10 / 3016065-03 \$ 15.00 / 0$
}

Many studies have attempted to lesion parts of the cerebellum to delineate functional roles to particular regions. That different regions are to an extent functionally distinct was first suggested by Chambers and Sprague (1955), who determined the classes of motor deficits that occurred after specific cerebellar cortical lesions. However, because individual cerebellar modules are narrow, ablations are likely to involve multiple modules. An alternate approach has been to eliminate the input to cerebellar modules, i.e., the inferior olive. This approach was used by Horn and colleagues (2010) in a recent paper in The Journal of Neuroscience.

The authors inactivated specific olivary subdivisions in cats with the glutamatergic receptor blocker CNQX. They first determined the extent of the spread and duration of CNQX inactivation by pressure injecting the blocker into the rostral dorsal accessory olive (rDAO), which has a well defined spatial map and is exquisitely sensitive to somatosensory stimulation, and recording spontaneous and peripherally evoked olivary activity. Using this technique, Horn and colleagues (2010) established that a $250 \mathrm{nl}$ injection inactivated a radius of $\sim 0.4-1.0 \mathrm{~mm}$ within the olivary target. Most olive regions relating to a given module are orientated into columns that are longer in the rostrocaudal axis. In the cat, the dimensions of olivary subdivisions that project to cerebellar modules vary between 350 and $500 \mu \mathrm{m}$ wide mediolaterally and $\sim 2.5 \mathrm{~mm}$ in the rostrocaudal extent (Armstrong et al., 1982; Trott and Apps, 1991). Although the size of the inactivation radius by Horn et al. (2010) is within the dimensions of an olivary module rostrocaudally, the area is twice the size mediolaterally. Therefore, the interpretation of chemical inactivation experiments requires caution since the injectate is unlikely to be confined exclusively to a specific region of a complexshaped nucleus such as the inferior olive without disturbing neighboring regions that target different cerebellar modules, particularly if injections are located near the boundaries of two cerebellar modules.

Next, the authors inactivated various subdivisions of the inferior olive and examined the behavioral deficits during a reach-to-grasp task and locomotion. Although inactivation of the rDAO produced no change in the trajectory of the contralateral limb during the reach-tograsp task, cats had difficulty grasping the handle that allowed them to retrieve their food reward. Multiple attempts were required to grasp the handles and when they managed to do so, the strategy they used was altered. In control groups, cats used their toes; after CNQX injections, they hooked their wrist around the handle (Horn et al., 2010, their Figs. 5A, 6A). The contralateral paw was also affected by CNQX injection during locomotion; the dorsum of the paw turned under and dragged along the floor, and the height of the paw was significantly reduced during the swing phase of the step cycle (Horn et al., 2010, their Figs. $6 B, 10 A$ ).

These findings differ from those in a recent study (Pijpers et al., 2008) that used an alternative approach in rats. Instead of chemically inactivating the olive, these authors cortically injected the retrogradely 
transported neurotoxin cholera toxin b conjugated to saporin to induce selective dysfunction of a complete module without affecting neighboring modules. Pijpers et al. (2008) demonstrated that selective impairment of the $\mathrm{C} 1$ hindlimb module in the rat did not lead to any recognizable defects during locomotion. However, the step phase-dependent modulation of cutaneously induced reflexes during locomotion was significantly reduced (Pijpers et al., 2008, their Fig. 7B) compared with that in control animals.

It is unlikely that the difference between the two studies solely results from a species difference topographically, as cerebellar modules are highly conserved across mammalian species (Apps and Hawkes, 2009). An alternative explanation is that the injections of CNQX into rDAO by Horn et al. (2010) disrupted more than a single cerebellar module. Three different modules (C1, C3, and Y) receive climbing fiber input from $\mathrm{rDAO}$. The cortical components of these modules are the $\mathrm{C} 1, \mathrm{C} 3$, and $\mathrm{Y}$ zones, which project to nucleus interpositus anterior (NIA). The three zones are thought to be functionally coupled because their corticonuclear output converge onto a common group of nuclear neurons in the NIA (Trott and Armstrong, 1987) and the three zones share climbing fiber inputs, some of which branch to innervate two of these zones (Ekerot and Larson, 1982). Moreover, it is likely that the complex topography of the inferior olive makes it difficult to isolate one region from another, especially the transition between DAO and principal olive (PO). The injection site diameter of $1 \mathrm{~mm}$ reported by Horn et al. (2010) should be sufficient for the blocker to spread to other parts of the olive, e.g., the PO, which target D modules and are thought to have a role in visually guided movements.

Horn et al. (2010) also examined the affects of CNQX injection in other cerebellar modules by injecting CNQX into the caudal and rostral medial accessory olives (cMAO and rMAO, respectively). The rMAO solely targets the C2 module, whereas cMAO is a composite nucleus composed of three subnuclei, which are associated with the A, AX, and A2 modules. Injections into cMAO can therefore affect all three of these modules. The role of the $\mathrm{C} 2$ module remains poorly understood, but it is thought to contribute to head orientation and gaze control, as well as voluntary limb movements. In contrast, the A modules are thought to be involved in the regulation of posture and balance (for review, see Cerminara and Apps, 2010). Horn et al. (2010) found that injections of CNQX into cat cMAO, which target the A modules, severely impaired balance during locomotion with little or no affect on voluntary limb movements during the reach-to-grasp task (Horn et al., 2010, their Figs. 5D, 14A). In contrast, rMAO injections, which target the C2 module, produced variable trajectories during the reach-to-grasp task, with the cats' forepaws frequently ending too low to grasp the handle to retrieve the reward (Horn et al., 2010, their Fig. 11). rMAO injections also severely disrupted axial support and, during locomotion, animals either limped with one or both contralateral limbs or walked with a waddling motion (Horn et al., 2010, their Fig. 12). Paw height during the step cycle was also significantly reduced (Horn et al., 2010, their Fig. 10 B). In sum, the behavioral effects are somewhat surprising given the roles afforded to the C2 (voluntary limb control) and A modules (posture and balance) and appear to be greater when CNQX was injected into the olivary subdivision that targets the $\mathrm{C} 2$ module rather than the A modules.

Using two different neurotoxins to selectively preserve the olivary subdivisions of either the A or C2 modules, Seoane et al. (2005) also investigated the role of these two modules in rats and demonstrated that motor deficits during beam walking, locomotion, and a vertical hold task were greater in rats treated with a toxin that kept the $\mathrm{C} 2$ module intact compared with rats treated with a toxin that preserved the A modules (Seoane et al., 2005, their Figs. 5, 6). This is presumably because the integrity of the A modules in animals treated with a toxin that preserved the A modules affords greater control of axial musculature that underpins most of the behavioral tests examined. This is contrary to the severity of the behavioral effects seen by Horn et al. (2010), who found that the behavioral deficits were greater when the $\mathrm{C} 2$ module was lost compared with the A modules. What could account for the difference between the two studies? Given that Horn et al. (2010) found that rMAO injections also severely disrupted axial support, the simplest explanation is that the injections centered on rMAO (C2 module) spread to include other subdivisions of the inferior olive, including those involved in posture (cMAO, A modules) and visually guided movements ( $\mathrm{D}$ modules).

A finding that Horn and colleagues (2010) did not comment on was an in- crease in both spontaneous and evoked olivary activity following injection of CNQX into rDAO (Horn et al., 2010, their Fig. $2 B$ ). This could have resulted from release from mutual inhibition between olivary cells (Andersson, 1984) whereby local CNQX injection could cause a general increase in inferior olive activity. Removal of the olivocerebellar climbing fiber projection either acutely or chronically has a profound influence on Purkinje cell activity, causing simple spikes to exhibit highly abnormal firing patterns (Cerminara and Rawson, 2004), whereas an increase in climbing fiber activity decreases Purkinje cell simple spike activity (Rawson and Tilokskulchai, 1982). Although Horn et al. (2010) emphasized the importance of the olivocerebellar circuit in regulating simple spike activity to maintain the operational state of the cerebellum for ongoing motor control, the loss of mutual inhibition was not considered. There are also various other network connections that have the potential to link the operation of different modules. These include electronic coupling within the olive, the collateral branching in the transverse axis of some climbing fibers and mossy fibers, the orientation and extent of parallel fibers, and the nonreciprocal organization of the nucleocortical projection (Apps and Garwicz, 2005; Bengtsson and Hesslow, 2006; Apps and Hawkes, 2009; Ruigrok, 2010).

Thus, although an individual module may play an important role in one particular aspect of motor control, it seems likely that individual modules interact with one another and may function as composite entities. Based on the available evidence, it therefore seems premature to conclude, as did Horn et al. (2010), that “. . . each cerebellar module has a specific and unique function in sensory-motor integration." Further studies are required to clarify this important issue.

\section{References}

Andersson G (1984) Mutual inhibition between olivary cell groups projecting to different cerebellar microzones in the cat. Exp Brain Res 54:293-303.

Apps R, Garwicz M (2005) Anatomical and physiological foundations of cerebellar information processing. Nat Rev Neurosci 6:297-311.

Apps R, Hawkes R (2009) Cerebellar cortical organization: a one-map hypothesis. Nat Rev Neurosci 10:670-681.

Armstrong DM, Campbell NC, Edgley SA, Schild RF, Trott JR (1982) Investigations of the olivocerebellar and spino-olivary pathways. In: The cerebellum: new vistas (Chan Palay V, Palay SL, eds.), pp. 195-232. Berlin: Springer. 
Bengtsson F, Hesslow G (2006) Cerebellar control of the inferior olive. Cerebellum 5:7-14

Cerminara NL, Apps R (2010) Behavioural significance of cerebellar modules. Cerebellum. Advance online publication. Retrieved September 14, 2010. doi:10.1007/s12311-010-0209-2.

Cerminara NL, Rawson JA (2004) Evidence that climbing fibers control an intrinsic spike generator in cerebellar Purkinje cells. J Neurosci 24:4510-4517.

Chambers WW, Sprague JM (1955) Functional localization in the cerebellum. I. Organization in longitudinal cortico-nuclear zones and their contribution to the control of posture, both extrapyramidal and pyramidal. J Comp Neurol 103:105-129.

Ekerot CF, Larson B (1982) Branching of olivary axons to innervate pairs of sagittal zones in the cerebellar anterior lobe of the cat. Exp Brain Res 48:185-198.

Horn KM, Pong M, Gibson AR (2010) Functional relations of cerebellar modules of the cat. J Neurosci 30:9411-9423.

Pijpers A, Winkelman BH, Bronsing R, Ruigrok TJ (2008) Selective impairment of the cerebellar $\mathrm{C} 1$ module involved in rat hind limb control reduces step-dependent modulation of cutaneous reflexes. J Neurosci 28: 2179-2189.

Rawson JA, Tilokskulchai K (1982) Climbing fiber modification of cerebellar Purkinje cell responses to parallel fiber inputs. Brain Res 237:492-497.

Ruigrok, TJ (2010) Ins and outs of cerebellar modules. Cerebellum. Advance online pub- lication. Retrieved March 16, 2010. doi:10. 1007/s12311-010-0164-y.

Seoane A, Apps R, Balbuena E, Herrero L, Llorens J (2005) Differential effects of trans-crotononitrile and 3-acetylpyridine on inferior olive integrity and behavioural performance in the rat. Eur J Neurosci 22:880-894.

Trott JR, Apps R (1991) Lateral and medial subdivisions within the olivocerebellar zones of the paravermal cortex in lobule $\mathrm{Vb} / \mathrm{c}$ of the cat anterior lobe. Exp Brain Res 87:126-140.

Trott JR, Armstrong DM (1987) The cerebellar corticonuclear projection from lobule $\mathrm{Vb} / \mathrm{c}$ of the cat anterior lobe: a combined electrophysiological and autoradiographic study. I. Projections from the intermediate region. Exp Brain Res 66:318-338. 